\title{
Leucocyte esterase determination as a secondary procedure for urine screening
}

\author{
PM LEIGHTON, JA LITTLE \\ From the Section of Microbiology, Dr Everett Chalmers Hospital, Fredericton, NB, Canada E3B 5N5
}

SUMMARY The use of a dipstick to detect leucocyte esteraseuria (Chemstrip L) was compared with the visualisation of leucocytes in a Gram stained smear for the detection of pyuria. The sensitivity and specificity of the two systems using the predictive value theory were similar. The use of a dual screening procedure (automation plus dipstick) allowed reliable $4 \mathrm{~h}$ screening of urine specimens for the detection of urinary tract infections.

Urine specimens comprise a major portion of the work of a clinical microbiology laboratory, and increasingly they are being examined by means of automated or semiautomated instruments. Many of these instruments are based on the principle that growth resulting from the inoculation of a broth medium with urine possessing significant bacteriuria $\left(10^{8}\right.$ organisms $/ 1,10^{5}$ organisms $\left./ \mathrm{ml}\right)$ will yield a specific change in the amount of transmitted light activating a photosensor. In this way urine specimens may be rapidly screened for the presence of significant numbers of bacteria, and only those specimens likely to show the presence of an infection need to be cultured for further examination.

There are two difficulties with this assumption. Firstly, it is now known that a urinary tract infection may involve fewer than $10^{8}$ organisms/l. Secondly, slow growing organisms may be present in the urine in significant numbers but may cause insufficient change in the transmitted light to be detected by the instrument. One way to circumvent these problems is to examine the urine specimen for the presence of leucocytes since pyuria is a reliable indicator of an inflammatory response in the urinary tract. ${ }^{12}$ This paper examines the use of the dipstick for leucocyte esterase as an alternative to the more time consuming Gram stained smear to detect the presence of leucocytes in the urine.

\section{Material and methods}

GRAM STAINED SMEAR

One drop of uncentrifuged urine (about $10 \mu \mathrm{l}$ ) was applied to a glass microscope slide using a bac-

Accepted for publication 22 October 1984 teriological loop. Two specimens were applied to each slide. The drops were air dried without spreading, stained, and examined under the low power objective $(\times 10)$. The number of leucocytes per low power field was recorded. When tabulating the results, counts of three leucocytes per low power field or less were treated as negative.

\section{LEUCOCYTE ESTERASE}

Leucocyte esterase was detected using the Chemstrip L dipstick (Boehringer Mannheim Canada Ltd, Dorval, PQ, Canada). The urine was brought to room temperature and the Chemstrip $L$ dipped into it for $1 \mathrm{~s}$. The excess urine was removed against the side of the container and the dipstick left in the air for $15 \mathrm{~min}$. The presence of leucocyte esterase was indicated by the development of a blue colour due to the enzymatic breakdown of an indoxyl ester and the subsequent oxidation of the indoxyl. A colour chart on the container indicated the colours resulting from a negative result, a trace of the enzyme, and a positive result.

\section{CULTURE}

Urine specimens were screened by means of the Autobac MTS (General Diagnostics, Scarborough, Ontario, Canada). Those specimens showing the presence of significant bacteriuria by the screen or the presence of bacteria and leucocytes in a Gram stained smear were cultured quantitatively. The urine was streaked on to trypticase soy agar with $5 \%$ sheep blood using a plastic disposable $1 \mu$ l loop (Nunc, Gibco Canada Inc, Burlington, Ontario, Canada), and the colony count was assessed the next morning. A positive urine was one with a count of more than $10^{8}$ organisms $/ 1$ for one or two organisms 
or more than $5 \times 10^{7}$ organisms $/ 1 \quad(50000$ organisms $/ \mathrm{ml}$ ) for a single organism.

\section{URINE SPECIMENS}

The study was divided into two parts. The first part compared the ability of the dipstick and the Gram stained smear to detect the presence of leucocytes in fresh specimens (less than $4 \mathrm{~h}$ old). A total of 481 urine specimens were examined, of which six were bloody and were omitted to avoid experimental bias. The specimens were refrigerated until examined, and then brought to room temperature before testing with the dipstick and preparing the smear. These results were then compared with the culture results obtained in the routine laboratory. Each of these steps (smear, dipstick, and culture) was performed by a different person unaware of the other results, and the data were compiled at the end.

The second part of the study concerned 510 unselected urine specimens, many of which were more than $4 \mathrm{~h}$ old at the time of examination. This provided a comparison of the two methods under actual laboratory conditions. The urine specimens were tested with the Chemstrip $L$ and the results compared with those from the Gram stained smear read in the routine laboratory. Since the technologists report the results semi-quantitatively, and in the light of the results in the first part of the study, a $+/-$ on the smear result (which approximated fewer than three leucocytes per low power field) was counted as negative in the tabulation of the results. Similarly, trace results with the Chemstrip L were counted as negative.

\section{STATISTICAL ANALYSIS}

Sensitivity, specificity, and predictive values were calculated using the predictive value theory. ${ }^{3}$

\section{Results}

The results for the first part of the study comparing the ability of the Gram stained smear and the dip-

Table 1 Comparison of the smear and the dipstick for the detection of pyuria and their correlation with culture results

\begin{tabular}{lllc}
\hline \multirow{2}{*}{ Leucocyte esterase } & \multicolumn{2}{l}{ Gram stained smear } \\
\cline { 3 - 4 } & & Positive $^{*}$ & Negative \\
\hline+ & Culture + & 53 & 8 \\
& Culture - & 25 & 22 \\
Trace & Culture + & 7 & 5 \\
& Culture - & 8 & 23 \\
- & Culture + & 3 & 22 \\
& Culture - & 15 & 284 \\
\hline
\end{tabular}

*More than three leucocytes per low power field $(\times 10$ objective $)$.
Table 2 Correlation of smear and dipstick methods for the detection of pyuria

\begin{tabular}{|c|c|c|}
\hline \multirow[t]{2}{*}{ Leucocyte esterase } & \multicolumn{2}{|c|}{ Gram stained smear } \\
\hline & Positive & Negative $\dagger$ \\
\hline $\begin{array}{l}\text { Positive } \\
\text { Negative* }\end{array}$ & $\begin{array}{l}84(16 \%) \\
25(5 \%)\end{array}$ & $\begin{array}{c}27(5 \%) \\
374(73 \%)\end{array}$ \\
\hline
\end{tabular}

*Trace results counted as negative.

$++1-$ result - that is, fewer than three leucocytes per low power $\overrightarrow{0}$ field ( $\times 10$ objective) counted as negative.

stick to detect pyuria are shown in Table 1. Pyuria was detected by both the dipstick and the smear in? 78 specimens $(16 \%)$, of which 53 yielded culture $\omega_{\infty}^{\omega}$ results indicative of a urinary tract infection. Both i systems showed the absence of pyuria in 306 speci- $N$ mens $(64 \%)$, of which 22 were subsequently found to have significant bacterial growth when cultured. $\triangle$ A total of 43 specimens ( $9 \%$ ) showed a trace-that $\vec{T}$ is, a weak reaction-with the leucocyte esterase test $\mathbb{Q}$ and 15 of these showed leucocytes in the stained smear. Only 12 of the 43 specimens yielded appreciable growth on culture. There were 18 specimens $(4 \%)$ which were negative for leucocyte esterase but $ळ$ were reported as showing leucocytes in the stained 9 smear, and three of these showed significant growth on culture. Thirty urine specimens $(6 \%)$ were leucocyte esterase positive even though no leucocytes were seen in the smear, and eight were confirmed on culture.

Table 2 shows the results of the second part of the $\stackrel{2}{\vec{F}}$ study. In $90 \%$ of the cases the smear and dipstick results agreed. Twenty seven specimens were $\bar{\supset}$ enzyme positive and smear negative and seven of these showed positive culture results. Twenty two specimens showed the converse (enzyme negative? but smear positive), and 10 of these were confirmed on culture.

\section{Discussion}

This laboratory possesses a General Diagnostics $\frac{D}{\square}$ Autobac MTS for use in urine screening. Growth resulting from a bacterial density in the urine of $10^{8} \mathrm{~N}$ organisms $/ 1$ (10 organisms $/ \mathrm{ml})$ causes a 0.2 ve decrease in the electrical output of the photodetec- 0 tor. Since bacterial growth rates vary, the incubation $\omega$ time for the urine and broth cultures must maintain? a balance between the detection of slow growing organisms and false positives due to small numbers $\Phi$ of organisms growing up during long incubation + periods. Whereas the percentage of positive urines $\frac{T}{T}$ detected increased from $88.8 \%$ at $3 \mathrm{~h}$ to $97.3 \%$ at $\frac{\mathrm{O}}{\mathrm{D}}$ $6 \mathrm{~h}$ the rate of false positives increased over the $\stackrel{\square}{\mathscr{P}}$ same period from $1.9 \%$ to $14.6 \% .^{4}$ In another $\stackrel{\Phi}{\varrho}$ study, Kelly and Balfour ${ }^{5}$ found that by reducing the 
incubation time to $4 \mathrm{~h}$ false positives were reduced to $53 \%$ of the specimens containing $10^{7}-10^{8}$ organisms/l and to only $6 \%$ of the specimens with less than $10^{7}$ organisms/l. At the same time, however, many Gram positive cocci, yeasts, and some strains of Pseudomonas aeruginosa grew too slowly to be detected even after 5 or $6 \mathrm{~h}$ incubation. ${ }^{4}$ These specimens would tend to be reported erroneously as showing no significant bacteriuria. The problem is complicated by the fact that bacterial concentrations in the urine of less than $10^{8}$ organisms $/ 1$ may, on occasion, be significant, and these must be differentiated from urethral contamination.

In order to overcome these difficulties, the laboratory has adopted a two pronged approach. The urine and broth cultures are incubated for only $4 \mathrm{~h}$ to minimise the number of false positive results, and Gram stained smears are examined to detect potentially true positive specimens missed by this short incubation. Gram stained smears are time consuming to prepare and read, and a faster method of detecting urinary tract infections due to slow growing organisms or smaller numbers of bacteria was sought. Working on the assumption that a urinary tract infection is likely to show pyuria as well as bacteriuria, we compared the results obtained by means of the Gram stain with the detection of leucocytes in the urine using a dipstick (Chemstrip L) to detect leucocyte esteraseuria. No attempt was made to differentiate specimens from male and female patients, but it has been previously reported ${ }^{6}$ that the test for leucocyte esterase is more reliable with male patients owing to the absence of contamination from other sources.

The dipstick for leucocyte esterase was as efficient as the Gram stained smear for detecting the presence of leucocytes in the urine. The smear detected leucocytes in 111 fresh urine specimens compared with 108 with the dipstick. In both cases almost $57 \%$ of these specimens were confirmed by culture to have significant bacteriuria. Similar results were obtained when there was no specimen selection and the study was not restricted to fresh specimens.

The dipstick indicated a trace amount of leucocyte esterase in 43 instances. Fifteen of them also showed leucocytes in the Gram stained smear and about half of these were confirmed by culture. Twenty eight urine specimens showing a trace for the enzyme reaction were negative with the smear, and only $18 \%$ of these showed significant bacteriuria on culture.

Previous studies $^{27}$ have compared the Chemstrip $L$ with chamber counts of leucocytes in urines and have obtained sensitivities of $88-95 \%$ and specificities of $94-98 \%$. This confirms the accuracy of the dipstick in detecting the presence of leuco- cytes. Similarly, this study found that the dipstick correlated well with the Gram stain for the detection of pyuria. When the Gram stain and dipstick results are assessed using the culture results as a standard they have sensitivities of $64 \%$ and $62 \%$ respectively, and both have a specificity of $87 \%$. They also had the same predictive values of $57 \%$ (positive predictive value) and $90 \%$ (negative predictive value). The positive predictive value agrees well with the correlation coefficient of 0.58 obtained by Kusumi $e t$ $a l^{7}$ between bacterial count and esterase test results.

Because this is a regional laboratory many specimens are not cultured within $4 \mathrm{~h}$ of specimen collection and reach the laboratory in refrigerated boxes. It might be expected that some of the leucocytes would lyse during transport so that they would not be detected on the Gram stained smear. Since the Chemstrip $L$ is not affected by the integrity of the cell, it might show the presence of leucocyte esterase where no leucocytes were seen. Table 2 shows that this did not occur and both systems were equally efficient at detecting pyuria in unselected specimens received in the laboratory.

Although at $160(9 p)$ each the dipstick is more expensive than the material used for a Gram stained smear ( $4 \notin$ or $2 p)$, there is a considerable saving in the cost of technologist time. The dipstick takes only a few seconds to perform, whereas the preparation, staining, and reading of a Gram stained smear may be expected to take 2-3 min. At present salaries this entails a cost of $40-60 e(22-34 p)$ per specimen, which more than compensates for the extra cost of the dipstick.

This investigation was carried out in order to develop an alternative, less time consuming method to Gram stained smears for the screening of urine specimens for pyuria. Complete reliance on automated urine screening methods yields either an unacceptably high rate of false positive results, or a failure to detect infections due to slow growing organisms or numbers less than $10^{8}$ organisms $/ 1$. For this reason it is desirable to supplement the automated urine screen with a rapid screen examining a different parameter. In view of the fact that leucocyturia is an important sign of inflammation, ${ }^{2}$ the detection of leucocytes in a Gram stained smear seemed to be a suitable procedure. This method has, in fact, been advocated as a screening procedure on its own.$^{8-10}$ It is time consuming, however, and the dipstick for leucocyte esterase was examined as an alternative method. It showed good correlation with the Gram stain results and possessed similar sensitivity and specificity when compared with the culture results, while being faster and easier to perform. By supplementing the automated urine screen with such a secondary screening system the reliability of the 
screening procedure is increased and results may be reported after $4 \mathrm{~h}$ with a high degree of confidence.

We thank Dr K Aterman and Dr L Bocci for their helpful advice and Mrs Margaret Fredericks for the preparation of this manuscript.

\section{References}

' Stamm WE. Measurement of pyuria and its relation to bacteriuria. Am J Med 1983;75(1B): 53-8.

${ }^{2}$ Gillenwater JY. Detection of urinary leukocytes by Chemstrip L. J Urol 1981;125:383-4.

${ }^{3}$ Ransokoff DF, Feinstein AR. Problems of spectrum and bias in evaluating the efficacy of diagnostic tests. $N$ Engl $J$ Med 1978;299:926-30.

${ }^{4}$ Hale DC, Wright DN, McKie JE, Isenberg HD, Jenkins RD, Matson JM. Rapid screening for bacteriuria by light scatter photometry (Autobac): a collaborative study. J Clin Microbiol 1981;13:147-50.
${ }^{5}$ Kelly MT, Balfour LC. Evaluation and optimization of urine screening by Autobac. J Clin Microbiol 1981;13:677-80.

- Perry JL, Matthews JS, Weesner DE. Evaluation of leukocyte esterase activity as a rapid screening technique for bacteriuria. $J$ Clin Microbiol 1982;15:852-4.

' Kusumi RK, Grover PJ, Kunin CM. Rapid detection of pyuria by leukocyte esterase activity. JAMA 1981;245:1653-5.

8 Pezzlo MT, Tan GL, Peterson EM, de la Maza LM. Screening of urine cultures by three automated systems. J Clin Microbiol 1982; 15:468-74.

" Pollock HM. Laboratory techniques for detection of urinary tract infections and assessment of value. Am J Med 1983;75 (1B):79-84 .

${ }^{10}$ Pfaller MA, Baum CA, Niles AC, Murray PR. Clinical laboratory evaluation of a urine screening device. J Clin Microbiol 1983; 18:674-9.

Requests for reprints to: Dr PM Leighton, Section of Microbiology, Dr Everett Chalmers Hospital, Priestman Street, Fredericton, NB, Canada E3B 5N5. 